\title{
Tracking Grade 10 learners' geometric reasoning through folding back
}

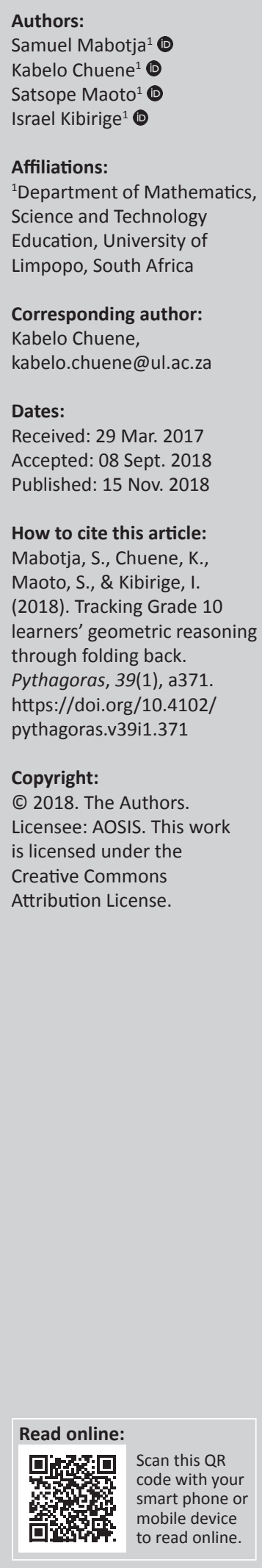

This article uses parts of qualitative data from the first author's study that focused on exploring Pirie and Kieren's process of folding back, revisiting previously held understandings of a concept for its extended understanding in order to solve the problem of enhancing Grade 10 learners' reasoning in geometry. In South Africa, secondary school leavers and pre-service teachers have difficulties in understanding geometric concepts and little is known about learners' geometric reasoning. Using teaching experiment methodology, we particularly tracked Grade 10 learners' geometric reasoning through folding back. We targeted two groups of learners from a class of 54 in which learners grouped themselves: the first group consisted of two members and the second group consisted of three members. The selection of learners was prompted by the manner in which the learners argued and questioned each other during four weeks of exploratory teaching. Data were collected through participant observations, where notes were taken and video recordings made in different sessions. We analysed the field notes and verbatim transcriptions of the video recordings using Polkinghorne's narrative analysis and analysis of narratives. Both the teacher's and the learners' interventions prompted effective folding back resulting in critical sharing of ideas. We thus argue that learners' effective folding back is a powerful tool to enhance their geometric reasoning.

\section{Introduction}

A few studies have focused on geometric reasoning (Battista, 2007; Budi, 2011; Evans, 2007; National Council of Teachers of Mathematics, 2002) to develop skills of explaining, arguing, verifying (Battista, 2007; Budi, 2011) and to relate problem-solving to mathematical proof (Evans, 2007). Thus, geometric reasoning needs to be developed to enable learners to process mathematical (geometric) proof (Evans, 2007). Proof is defined as a chain of deductions through which the truth of the propositions to be proved is derived from axioms and previously established propositions (Fetisov \& Dubnov, 2012). Briefly, proof consists of explicit chains of inference following agreed rules of deduction (Hanna \& De Villiers, 2008). Similarly, Brown, Jones, Taylor and Hirst (2004) contend that geometric reasoning is important in developing and evaluating deductive arguments about figures and their properties that help learners make sense of geometric situations. Emphasis on geometric reasoning can help learners organise their mathematical knowledge in ways that enhance their conceptual development in geometry (National Council of Teachers of Mathematics, 2002). Learning procedures and proofs without a good understanding of geometric concepts may leave learners ill-equipped for geometric reasoning (Department of Basic Education, 2011). Thus, it could be argued that learners should not only develop conceptual understanding of geometric properties of figures, but also be able to apply such properties to solve geometry problems. Pirie and Kieran (1994) explain this through their theory of growth in mathematical understanding that comprises eight nested levels. Named in their succeeding order from the inner layer these levels are: primitive knowing, image making, image having, property noticing, formalising, observing, structuring and inventising. It is in these levels where Pirie and Kieran introduce the concept of folding back. Folding back is a non-unidirectional process through which mathematical understanding grows through these levels. Therefore it is a cognitive shift that happens when previously held understanding, inner layer understanding, acts are informed by outer layer knowing that ultimately leads to further outer layer understanding (Pirie \& Martin, 2000). There are few studies that show that folding back extends learners' existing understanding of mathematics concepts (Delgado, Codes, Monterrubio, \& Astudillo, 2014; Martin \& Towers, 2016; Wright, 2014). Using Tall's (2008) argument, learners' geometric development builds on experiences that they have met before. Previous experiences, referred to as met-befores, form connections in the brain and that would affect how learners make sense of new mathematical situations that are either consistent (Martin \& Towers, 2016) or inconsistent (Tall, 2008). 
Nevertheless, there are difficulties learners encounter in geometry (Fujita, Yutaka, Kunimune, \& Jones, 2014; Gunhan, 2014; Naidoo, 2013). For instance, Fujita et al. (2014) found that, although learners discovered various methods to construct a square, they had difficulties in using properties of shapes to find out why their construction would be correct. Their findings further indicated that learners rely on visual perception of the given geometrical shape rather than deduction based on the properties of the geometrical object. Similarly, Gal and Linchevski (2010) confirmed that children prefer to rely on a visual prototype, rather than a verbal definition, when classifying and identifying shapes. The results of Gunhan's (2014) study showed that learners sometimes have insufficient geometrical knowledge and visual perception and, at times, are not able to provide a mathematical argument. He recommended that the school curriculum should place more emphasis on reasoning skills and that learners should be presented with problems in order to apply different reasoning skills. In South Africa, secondary school leavers (Panaoura \& Gagatsis, 2009) and pre-service teachers (Ndlovu \& Mji, 2012) were reported to have difficulties in understanding geometric concepts. In view of these learning difficulties, this study aimed at tracking learners' geometric reasoning through folding back. The findings from this study may contribute to the existing knowledge regarding teacher-learner interactions and the learners' growth of geometrical understanding.

\section{Theoretical framework}

This article is guided by Pirie and Kieren's (1994) dynamical theory of the growth of mathematical understanding. This theory deals with the growth of mathematical understanding and comprises eight nested levels, namely primitive knowing, image making, image having, property noticing, formalising, observing, structuring and inventising. 'By "images" the theory means any ideas the learner may have about the topic, any "mental" representations, not just visual or pictorial ones' (Martin, LaCroix, \& Fownes, 2005, p. 22). Levels used in this study are explained as:

- Primitive knowing refers to the knowledge that learners bring to their learning context as prior knowledge.

- Image making is the level where a learner engages in activities intended for developing ideas and images for a concept (Martin et al., 2005).

- Image having is a layer where learners can use an understanding of a topic to help them to make mental plans that can be used when working on mathematical tasks (Martin et al., 2005).

- Property noticing deals with learners' examination of an image for relevant properties.

- Formalising is a level where learners consciously notice properties and work with them.

The nesting illustrates that the growth in mathematical understanding is neither linear nor unidirectional (Pirie \& Martin, 2000) and that each layer contains the preceding layer and is contained in the layer that follows (Pirie \&
Kieren, 1994). For Pirie and Kieren, this mutual inclusivity of layers symbolises back and forth movement of growth in understanding across these layers, a process they referred to as folding back. Although Martin (2008) agrees with Pirie and Kieren's definition of folding back, he is of the view that the definition 'remained essentially undeveloped and unelaborated in their work' (p. 64). According to Martin, how and why folding back occurs is not deeply explored. He thus expands the notion of folding back by providing three key elements: source of intervention, form of actions that leads to folding back and outcome of folding back. These elements were adopted to guide this study and are illustrated in Figure 1 and the accompanying narrative.

Each of the three elements explains some practice of folding back that allowed us to describe folding back and trace it to its source. Folding back may result from a teacher's intervention,



Source: Martin, L.C. (2008). Folding back and the dynamical growth of mathematical understanding: Elaborating the Pirie-Kieren theory. Journal of Mathematical Behavior, 27(1), 64-85 (p. 72). https://doi.org/10.1016/j.jmathb.2008.04.001

FIGURE 1: The framework for describing folding back. 
material intervention, peer intervention by another learner, or self-invoked intervention by a learner who decides to fold back. Depending on whether or not the source element is intended to result in folding back, the source of intervention can either be intentional or unintentional. An intentional intervention is said to be explicit or focused if it points directly to the idea, image or existing understanding; otherwise it is said to be unfocused.

The second of the three acts of folding back is the form of folding back. It has three categories that express how learners react to interventions by a source. This may take place according to four different categories, namely working at an inner layer using existing understanding, collecting at an inner layer to retrieve previous knowledge for a renewed view that is in line with what is needed for mathematical actions at hand, moving out of topic and working there, and causing discontinuity in the understanding that is developed. Working at an inner layer using existing understanding occurs when a learner is aware of limitations of their existing understanding at an outer layer and hence works in a less formal and less sophisticated way. This is done in such a way that less formal understandings are informed and assisted through formal understandings. Evidence of working at an inner layer using existing understanding is noticeable when learners either generate new ideas or change how they originally constructed a concept. On the other hand, the act of collecting at an inner layer happens with learners' sense of 'knowing that they know what they need to know', of being aware that they have the necessary understandings, but that they are just not immediately accessible (Martin, 2008, p. 77).

This knowledge and understandings are used to re-view and re-read concepts to meet the needs of the task at hand. Furthermore, folding back may involve stepping out of a topic to allow for development and thickening of another topic so that ultimately it enables working at an outer layer - a process referred to as moving out of topic and working there.

While working at an inner layer using existing understanding, collecting at an inner layer, and moving out of topic and working there result in thickening or further and enriched understanding of an existing concept, the act of causing a discontinuity in folding back does not. Causing a discontinuity takes place when interventions that cause cognitive shifts, referred to as invocative interventions, do not have an effect on understandings that are developed. This happens when a learner returns to an inner layer but fails to connect understandings that are developed with already existing ones.

The three forms of folding back that do not lead to a discontinuity result in two categories of outcomes of folding back. The outcome of folding back is either effective or ineffective. The outcome of folding back is effective if the result of folding back enables further understanding at an outer layer with or without external prompts. Otherwise the outcome of folding back is ineffective.

\section{Research methodology}

A teaching experiment methodology research design (Steffe \& Thompson, 2000), which researchers use to experience learners' mathematical learning and reasoning, was used to track whether folding back (Martin, 2008; Martin \& Towers, 2016; Pirie \& Kieren, 1994) supported Grade 10 learners' development of geometric reasoning. Using data from a master's study (Mabotja, 2017), we targeted data from two groups of learners from a class of 54 in which learners grouped themselves: the first group consisted of two members (John and Koena) and the second group consisted of three members (Lesiba, Lebogang and Sipho). These two groups were chosen because of the manner in which learners within the groups argued and questioned each other during the four weeks of exploratory teaching. Also, the groups were picked out because their arguments and questioning epitomised instances of folding back and not because folding back was normative among all groups. Data were collected through participant observations by the first author through field notes and video recordings of learners' conversations in different sessions.

Data collected were conversations between learners. In some instances the teacher was part of the conversations. The conversations were analysed through rewriting them as stories - narrative analysis (Polkinghorne, 1995). The stories were not descriptions of these conversations but interpretations and extrapolations of latent meanings so that the conversations could be coherently understood. Polkinghorne (1995) and Kim (2015) branded this as a smoothing process that allows for filling gaps in the story. This is done so that the story can be plotted into a coherent outcome while being aware that each group and thus each conversation was unique. At the same time, Martin's (2008) theory of folding back provided focus to how data were analysed. The three elements of the theory, namely the source of intervention, the form, and the outcome, were picked out of the conversations as relevant for interpreting the conversations. Thus aspects of analysis of narratives (Polkinghorne, 1995) were also used in analysing the conversations.

\section{Quality criteria}

To ensure quality criteria, transferability, confirmability, credibility and dependability were employed (Bitsch, 2005). For transferability, excerpts of conversations among learners provided the researchers with equivalents of descriptions in order to draw more information about data from the same context (Bitsch, 2005). Confirmability is the degree to which the findings can be confirmed by other people and can be traceable to the original data sources through the excerpts from which findings were drawn. In both instances, since the original data were captured on video, the learning scene could be revisited (Martin, 1999; Powell, Francisco, \& Maher, 2003). For credibility a prolonged engagement of about 30 days, and being on the research site a month prior to data collection, was used in 
order to overcome distortion of claims made and to increase subjectivity (Guba \& Lincoln, 1989). For dependability, diachronically reliable evidence was obtained through time triangulation (Cohen, Manion, \& Morrison, 2007). The extracts reported on in this article were a week apart from each other. In addition, monitoring of constructs was made by involving two of the authors in using Martin's (2008) framework of folding back to illustrate how the intervention, form and outcome were used in the study.

\section{Ethical considerations}

The participants and their parents completed the informed consent forms. Informed consent acknowledged the protection of the participants' rights (Creswell, 2012). For video recordings in the classroom and afternoon sessions, permission was requested from the school principal, the parents of the participants as well as the participants themselves. In order to ensure anonymity, pseudonyms were used for the participants and the research site.

\section{Findings and discussions}

We organised this section by drawing extracts from the main study's data and only tracking the two groups of students in order to demonstrate instances of conversation where folding back led to learners' geometric reasoning or not. According to Pirie and Martin (2000), brief extracts of dialogue are sufficient to draw attention to the shifts in thinking that took place. The data revealed that learners' critical sharing of ideas, and the teacher's intentional intervention, are critical elements for effective folding back. We started by capturing the learning activity that was given and thereafter analysed the learners' interactions by considering the source of the intervention, form and outcome of folding back (Martin, 2008).

\section{Extract 1: Prove that $\triangle P S T\|\| \triangle P Q R$}

The learners were given a learning activity to prove that $\triangle P S T$ | | | $\triangle P Q R$ (see Figure 2).

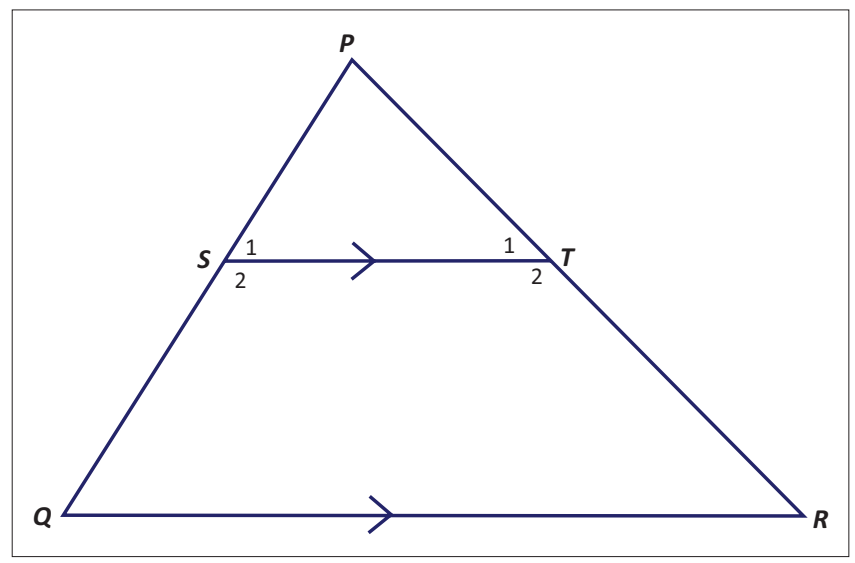

Source: Mabotja, K.S. (2017). An exploration of folding back in improving Grade 10 students reasoning in geometry. Unpublished master's thesis, University of Limpopo, Mankweng, South Africa (p. 73). Retrieved from http://hdl.handle.net/10386/1805

FIGURE 2: Corresponding angles of similar triangles.
Learners were expected to show that since lines $S T$ and $Q R$ are parallel, the corresponding angles of the two triangles are equal (Mabotja, 2017, p. 73):

\subsection{Koena: Here we can use PS and the other side?}

1.2 John: Look ... okay ... you cannot just say $P S$, so what are the values of PS? But we cannot use it if we are not given values. If we can determine angles of these triangles, then we can prove that these triangles are similar. So $\angle P$ is common, what about other angles? These are angles on a straight line; these are parallel lines. We need a transversal; we use those things of corresponding angles.

1.3 Koena: $\angle S_{1}$ is equal to $\angle Q$, as you see they form a $\mathrm{F}$ shape.

1.4 John: F shape this way?

[John traces an $F$ on the diagram with a pencil]

1.5 Koena: Yes.

1.6 John: Yes, it is correct.

1.7 Koena: If we can put it this way, can't we take it out?

[Koena rotates the worksheet as he says that the triangle is rotated $180^{\circ}$ ]

1.8 John: Yes, it is correct, they are this way, and then $\angle R$ is equal to $\angle T_{1}$. They are corresponding angles.

[John traces the F on the diagram with his finger]

1.9 John: Then we can say in triangle $P S T$ and triangle $P Q R$ [talking and writing], $\angle T_{1}$ is equal to $R$ [talking and writing].

1.10 Koena: Corresponding angles are equal.

[John and Koena simultaneously say angle $P$ is equal to angle $R$, common]

[John talking and writing]

1.11 Koena: $\angle S_{1}$ is equal to $\angle Q$.

1.12 John: They are corresponding angles.

1.13 John: Therefore, we can say triangle $P S T$ and $P Q R$ are similar.

1.14 Koena: Yes, they are similar.

[John and Koena simultaneously say angle, angle and angle] [John writes AAA]

The two learners in Extract 1 were given a task that required them to prove that the two triangles are similar. On the model for growth in mathematical understanding, this task is pitched at the level of formalising understanding in that it required learners to notice and work with the property of similar triangles that corresponding sides have equal ratios. Furthermore, for the learners to be able to provide the required proof, they should have the image that when two parallel lines are cut by a transversal, the resulting corresponding angles are congruent. The extract begins with Koena's intentional intervention that they work with side PS and another side. Such an utterance could have resulted from knowing that they had to prove that the ratio of $P S$ and its corresponding side is proportional to the other two corresponding sides of the triangles. However, John did not work at this level of the task to grow his understanding. Instead, as a form of folding back, he worked at an inner level 
of property noticing. From the given information on the task, he noticed that since the magnitudes of the sides of the triangles (and probably the proportionality between ratios of corresponding sides) were not known, it could not be argued that the two triangles were similar. He further uttered that 'if we can determine angles of these triangles, then we can prove that these triangles are similar'; thus suggesting an intentional intervention though not making it explicit. John did not explicitly indicate that the measures of two angles of one triangle should be equal to two corresponding angles of the other triangle for the two triangles to be similar.

All the same, from the given information of the task, John noticed that lines $S T$ and $Q R$ are parallel, and that they 'need $[e d]$ a transversal; we use those things of corresponding angles'. Here it can be claimed that John was aware that when parallel lines are crossed by a transversal, the corresponding angles are equal. At this stage Koena also worked at an inner layer using existing understanding as a form of folding back through uttering that $\angle S_{1}$ and $\angle Q$ form an $\mathrm{F}$ shape - a mnemonic used in most teaching to identify corresponding angles. This seemed to indicate that Koena needed the image of the ' $\mathrm{F}$ shape' mnemonic to notice that $\angle S_{1}$ and $\angle Q$ are corresponding angles. Working at property noticing John traced the ' $\mathrm{F}$ shape' and Koena rotated the diagram to have a clear picture of what they were talking about. The two learners seemed satisfied with their identification of two pairs of corresponding angles that are equal, namely $\angle T_{1}$ and $\angle R$ (see line 1.8), and $\angle S_{1}$ and $\angle Q$. After folding back to the property noticing level through using the F shape mnemonic and thickening their understanding through successfully applying the mnemonic on the given problem, the learners returned to the formalising layer without external prompts to conclude that the two triangles were similar. This was an instance where folding back was effective in geometric reasoning.

\section{Extract 2: Finding the magnitudes of $\boldsymbol{a}$ and $\boldsymbol{b}$}

In Extract 2 the learners must find the magnitudes of $a$ and $b$ in the given diagram (Figure 3).

Extract 2 shows an instance where moving out of topic and working there resulted in a discontinuity. With critical sharing of ideas on what caused the discontinuity, which emerged after Extract 2, folding back was ultimately effective. The extract was generated from the interaction between Lesiba and Lebogang on the learning activity on finding the magnitude of $a$ and $b$ after showing that triangles $\triangle T R S$ and $\triangle T P Q$ are similar. Although Sipho was a member of the group, he did not contribute actively during this discussion (Mabotja, 2017, pp. 76-79):

2.1 Lesiba: Here we can start by finding the sides that are in proportion.

2.2 Lebogang: Here we can separate the triangles [observed drawing $\triangle T R S$ and $\triangle T R S]$.

2.3 Lesiba: Yes, that is correct. Then we can say side $T R$ over side $T P$ is equal to $T S$ over $T Q$.



Source: Mabotja, K.S. (2017). An exploration of folding back in improving Grade 10 students reasoning in geometry. Unpublished master's thesis, University of Limpopo, Mankweng, South Africa (p. 75). Retrieved from http://hdl.handle.net/10386/1805

FIGURE 3: Proportionality in similar triangles.

2.4 Lebogang: Okay, it will be this way [observed writing $\left.\frac{T R}{T P}=\frac{T Q}{T S}\right]$. Side $T R$ is equal to 15 and $T Q$ is equal to 9.

2.5 Lesiba: Yes, let us continue.

2.6 Lebogang: We can say $a$ over 15 is equal to $b$ over 9 [observed writing $\frac{a}{15}=\frac{\frac{b}{4}}{9}$ ].

2.7 Lesiba: We can now cross multiply.

2.8 Lebogang: It is going to be 9 multiply $a$ is equal to $b$ is equal to 15 multiply $b$ over 4 [observed writing $9 a=15 \times \frac{b}{4}$ ]. Here $a$ is equal to eerrhhh ... two unknowns.

2.9 Lesiba: Two unknowns, how? [observed looking into the triangles Lebogang has drawn]

2.10 Lebogang: Yes $\left[9 a=15 \times \frac{b}{4} ; 9 a=\frac{b}{60} ; \frac{9 a}{9}=\frac{\frac{b}{60}}{9} ; a=\frac{20}{3} b\right]$

Similar to Extract 1, Extract 2 began with peer intervention by Lesiba. The intervention was intentional and explicit as he suggested that they find the sides of the triangles that were proportional. In order to do that Lebogang drew $\triangle T R S$ and $\triangle T P Q$ separately. In this case in particular, it can be claimed that Lebogang preferred working in a less formal way while working at an inner layer using existing understanding because she worked at an image making level. This was followed by an utterance by Lesiba that $\frac{T R}{T P}=\frac{T S}{T Q}$. Lebogang transcribed what Lesiba said incorrectly and wrote $\frac{T R}{T P}=\frac{T Q}{T S}$, instead of $\frac{T R}{T P}=\frac{T S}{T Q}$. Regardless of this error, it can be claimed that they intended to use their knowledge of proportionality between corresponding sides of two similar triangles in order to solve the given problem. Also shown in the extract is that the learners did not read the labelling of the diagram well. Instead of reading the magnitude of $T P$ and $T Q$ as $a+15$ and $\frac{b}{4}+9$ they read them as 15 and 9 respectively as evidenced 
when Lebogang drew the two triangles separately and labelled two sides. But when representing the equation $\frac{T R}{T P}=\frac{T Q}{T S}$ by the magnitude of the sides of the triangle they wrote $\frac{a}{15}=\frac{\frac{b}{4}}{9}$, which attests to the claim that they attempted to use proportionality of corresponding sides of similar triangles. There was a pause as Lesiba observed the two triangles that Lebogang drew, and also wanted clarity on why they ended with 'unknowns' $a$ and $b$ in $\frac{a}{15}=\frac{\frac{b}{4}}{9}$ (line 2.9). Regardless, Lebogang simplified the rational equation as $a=\frac{20}{3} b$ (line 2.10), an error that indicated that incorrect algebraic processes were followed. This led to a discontinuity that emanated from algebraic errors committed while working outside the topic of geometry.

The extract that follows continues Extract 2. It shows how the learners re-started the process of folding back after a discontinuity. It began after another pause in the learners' conversation and presumably thinking about what they could do next. It began with Lesiba's intentional intervention to show Lebogang that they did not read the measures of the sides of triangles correctly. Accordingly, this was an explicit intention (lines 2.11, 2.13 and 2.15) (Mabotja, 2017, p. 78):

2.11 Lesiba: Actually can't we have ... let me ask ... on TP can't we have $15+a$ ?

2.12 Lebogang: TP?

2.13 Lesiba: $\quad 15+a$ over $\ldots$ no $a$ over $15+a$ is equal to.

2.14 Lebogang: $a$ over 15 times $a$ ? [confused as to what to write]

2.15 Lesiba: [takes the book from Lebogang and writes $\frac{a}{15+a}$ ]

2.16 Lesiba: Therefore we say $a$ over $15+a$

[writes $\frac{a}{15+a}$ in his book] is equal to,

like here we say $9+b$ over 4 [writes $\frac{\frac{b}{4}}{\frac{b}{4}+9}$ ]

2.17. Lebogang: The whole of side of $T Q$ is equal to $b$.

The extract shows that, regardless of Lebogang's confusion in lines 2.2 and 2.4 of Extract 2, ultimately the students realised that $\frac{T R}{T P}=\frac{T S}{T Q}$ is equivalent to $\frac{a}{15+a}=\frac{\frac{b}{4}}{\frac{b}{4}+9}-$ this was a new way of understanding how the learners read the given information. With this new way of understanding the learners were able to fold back through the form of moving outside the topic of geometry and working on algebra as captured in the next extract (Mabotja, 2017, p. 80).

2.18 Lesiba: $\quad T R$ divided by $T P$ is equal to $T S$ divided by $T Q$ [writes $\frac{T R}{T P}=\frac{T S}{T Q}$ ]. TR is a divided by 15 plus $a$ is equal to $b$ divided by 4 divided by 9 plus $b$ divided by 4 .
2.19 Teacher: Yes, that equation is correct.

2.20 Lebogang: Cross multiply.

2.21 Lesiba: Cross multiply.

2.22 Teacher: Okay before we cross multiply.

2.23 Lesiba: We can say $b$ divided by 4 , this way [starts writing $\left.\frac{b}{4} \div 9+\frac{b}{4}\right]$

2.24 Teacher: Okay continue

2.25 Lebogang: Write $b$ divided by 4 , do we divide it?

2.26 Lesiba: Then we say times, then 4 goes on top ...

2.27 Lebogang: I don't understand you ...

2.28 Lesiba: You don't understand me, like, you see now is $\frac{b}{4} \div 9+\frac{b}{4}$, then we say $b$ divided by 4 , times $9+\frac{4}{b}\left[\right.$ writes $\left.\frac{b}{4} \times 9+\frac{4}{b}\right]$.

2.29 Lebogang: Yes, write them, but let us first replace $9+\frac{b}{4}$ with $b$.

2.30 Lesiba: $\quad$ Okay I see it since $9+\frac{b}{4}$ is equal to $b$

2.31 Lebogang: Yes this line here [pointing to the line outside the triangle] shows that $T Q$ is $b$.

2.32 Lesiba: Okay, then we will write it this way $\left[\right.$ writes $\left.\frac{T R}{T P}=\frac{T S}{T Q}\right]$.

2.33 Lebogang: Then we can start substituting.

2.34 Lesiba: Here is going to be this way [observed writing $\frac{a}{15+a}=\frac{\frac{b}{4}}{b}$ ], then this one [pointing to $\frac{\frac{b}{4}}{b}$ ]

we must change it.

2.35 Lebogang: Yes, we change it to $b$ over 4 divide by $b$ over 1 .

2.36 Lesiba:

Then it will be this way

[observed writing $\frac{a}{15+a}=\frac{b}{4} \div \frac{b}{1}$ ]

2.37 Lebogang: And then now we can change it by using multiplication.

2.38 Lesiba $\quad\left[\right.$ observed writing $\frac{a}{15+a}=\frac{b}{4} \times \frac{1}{b}$ ]

2.39 Lebogang: Here [pointing to $\frac{b}{4} \times \frac{1}{b}$ ] $b$ will multiply $b$.

2.40 Lesiba: Then this side [pointing to $\frac{b}{4} \times \frac{1}{b}$ ] with 1 over 4

$$
\text { [observed writing } \frac{a}{15+a}=\frac{1}{4} \text { ] }
$$

2.41 Lebogang: Then we can cross multiply.

The conversation began with Lesiba rewriting the equation $\frac{T R}{T P}=\frac{T S}{T Q}$ as its equivalent using the magnitudes of its sides while wrongly reading out what he wrote. The teacher acknowledged the equation written by Lesiba, $\frac{T R}{T P}=\frac{T S}{T Q}$, by uttering that that the equation was right. Lebogang intervenes intentionally by suggesting that they should 'cross multiply'. 
With this the learners continued working outside the topic of geometry. The teacher interrupted Lebogang's intervention by his utterance in line 2.22. Although the utterance was not explicit, it suggested to Lesiba that he had to rewrite $\frac{\frac{b}{4}}{9+\frac{b}{4}}$ as $\frac{b}{4} \times 9+\frac{4}{b}$, which was not correct. Lesiba was supposed to invert the entire compound factor $9+\frac{b}{4}$ so that $\frac{\frac{b}{4}}{9+\frac{b}{4}}$ was written as $\frac{b}{4} \times \frac{4}{36+b}$; instead he inverted only $\frac{b}{4}$ to be $\frac{4}{b}$. The learners did not correct the wrong representation but instead continued to work on it and substituted $9+\frac{b}{4}$ with $b$ as per Lebogang's suggestion. Although the substitution was syntactically wrong, it led to a correct representation of $\frac{T R}{T P}=\frac{T S}{T Q}$ as $\frac{a}{15+a}=\frac{1}{4}$. In this case, working outside the topic did not lead to a developed understanding of algebraic expressions which Martin (2008) would refer to as thickening. Nonetheless, following line 2.41 the learners were able to evaluate $a$ and $b$ as required in the given task, resulting in effective folding back.

It can thus be concluded that this was an instance where effective folding back happened regardless of a discontinuity as a form of folding back. Furthermore, it can be claimed that this effective folding back happened because of critical sharing of ideas and reflection. Algebraic errors that were made during folding back through working out of the topic, which did not result in a discontinuity, led to an effective folding back.

\section{Extract 3: Prove that $A B C D$ is a parallelogram}

In this activity learners were expected to prove from the given diagram (Figure 4) that quadrilateral $A B C D$ is a parallelogram, where $B F=A F$ and $C E=C D$ and the angle sizes are as shown.

Since the task required them to notice properties of the given diagram and to work with them to prove that $A B C D$ is a parallelogram, the diagram was pitched at a formalising level of understanding.

3.1 Lebogang: $\angle A$ is equal to $\angle C$, opposite angles of a parallelogram are equal.

3.2 Teacher: Which parallelogram are you looking at?

3.3 Lebogang: $A B C D$.

3.4 Teacher: And then what are you supposed to do?

3.5 Sipho: Prove that it is a parallelogram [image having].

3.6 Teacher: So, are you saying $\angle A$ is equal to $\angle C$ ? Or are you saying if you can find $\angle A$ being equal to $\angle C$, then you can conclude that $A B C D$ is a parallelogram?

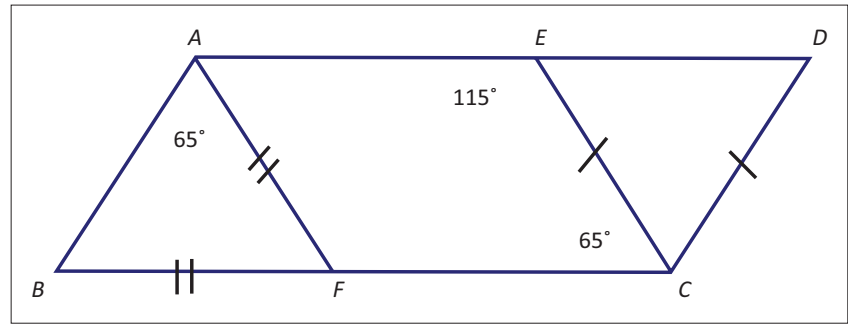

Source: Mabotja, K.S. (2017). An exploration of folding back in improving Grade 10 students reasoning in geometry. Unpublished master's thesis, University of Limpopo, Mankweng, South Africa (p. 97). Retrieved from http://hdl.handle.net/10386/1805

FIGURE 4: Proving a quadrilateral to be a parallelogram.

In this extract (Mabotja, 2017, p. 98) a peer, Lebogang, was the source of the intervention as she initiated the conversation. She intended for the group to notice that $\angle A$ is equal to $\angle C$ since $A B C D$ is a parallelogram. This could only be true if Lebogang meant that $\angle B A F$ and $\angle E C F$ are equal since this information was given. But since Lebogang did not use three letters, with the middle letter being the vertex, to read the angles it was not clear which angles $\angle A$ and $\angle C$ she talked about. This made her intervention unfocused. This could have been the reason why the teacher asked her to specify the parallelogram she was looking at. Since Lebogang was looking at quadrilateral $A B C D$, she used an invariant property of parallelograms that opposite angels are congruent. This could have happened because Lebogang did not intuitively differentiate between what was given and what was to be proved. Brown et al. (2004) asserted that this may result from using recently introduced ideas that are not well understood or a requisite to provide proof for something that is obvious.

Lebogang's confusion probably resulted in the teacher's intentional and explicit intervention. The teacher wanted Lebogang to specify what the task required her to do (line 3.4). This request could have made her realise that her intervention was unfocused. She did not respond; instead Sipho did. When the teacher wanted the learners to clarify if $\angle A$ and $\angle C$ were given as equal or whether the task required them to prove that these angles are equal (line 3.6), none of the learners responded. Instead, there was a significant pause during which the learners were probably critically considering the teacher's question. After this pause Lebogang did not pursue her initial intervention (line 3.7 below) thus resulting in a discontinuity at property noticing. It can be asserted that the discontinuity resulted because, using Pierie and Kieren's model of growth in understanding, Lebogang was at a primitive level, and the knowledge she had, that $\angle A$ is equal to $\angle C$ because they are opposite angles of a parallelogram(line 3.1), was insufficient for her understanding to grow.

The vignette that follows shows how folding back proceeded towards completion of the required proof (Mabotja, 2017, pp. 99-101).

\subsection{Lebogang: Is $\triangle A B F$ an isosceles triangle? \\ 3.8 Lesiba: Yes.}

3.9 Lebogang: Then it means ... we can say $\angle B$ is equal to $65^{\circ}$ ... base angles of iscosceles triangle are equal. 
3.10 Sipho:

Where is an isosceles triangle here?

3.11 Lebogang: Is not this $\triangle A B F$ an isosceles?

3.12 Lesiba:

Two sides are equal [pointing to the sides].

3.13 Teacher:

Yes that will be true.

3.14 Sipho:

Let us find all the angles, in that way we can see if it's a parallelogram.

3.15 Teacher: You are thinking of finding all the angles?

3.16 Sipho:

Yes ... and in that way we will see whether our opposite sides are equal or not, and if they are equal and then this means it is a parallelogram and if they are parallel.

3.17 Lebogang: How are we going to use the angles?

[Silence]

The extract above could have been triggered by the teacher's intervention when seeking clarification on what Lebogang had said. The peer intervention is explicit and intended to show that $\angle B$ equals $65^{\circ}$. Lebogang seemed to be at image having level of understanding since her utterance suggested she had mental constructs of isosceles triangles without first developing it. She then folds back to the primitive knowledge level and uses her existing knowledge on properties of isosceles triangles to come to a conclusion on the size of $\angle B$. This folding back was not effective for Sipho, who could not identify the isosceles triangle that Lebogang referred to. Once Lebogang pronounced triangle $\triangle A B F$ as the one she worked on, and once Lesiba showed Sipho the sides that were equal (line 3.12), Sipho seemed to understand what Lebogang was saying, achieving their folding back jointly. Sipho then suggested that the group find the sizes of the other angles of the quadrilateral in order to infer that the opposite angles of $A B C D$ were equal (line 3.14). Sipho showed traits of the property noticing level of understanding and of being aware of a rudimentary fact about parallelograms: that their opposite angles are equal. When the teacher asked if he intended to find the size of all angles (line 3.15), Sipho responded in the affirmative. It seems he suggested that they could apply their understanding of isosceles triangles to find the size of the opposite angles of quadrilateral $A B C D$ and further show that sides are equal to prove that the quadrilateral is a parallelogram (line 3.16). Although Sipho's suggestion was explicit, Lebogang did not know how to proceed with it. Hence her question: 'How are we going to use the angles?'

At this stage the learners could have either used the same procedure used for finding the size of $\angle B$ to find the size of $\angle D$ or show that $\angle B A D$ is equal to $\angle B C D$. The conversation that follows shows that, although Sipho suggested earlier that the size of $\angle C D A$ be found in the same way as the size of $\angle A B C$ the suggestion was not considered. Instead, in the conversation that follows, the teacher intervened explicitly with a different procedure to be followed (line 3.18). The intervention did not develop Sipho's growth in understanding as none of the people in the group allowed him to continue with his thinking, hence causing a discontinuity (Mabotja, 2017, pp. 99-101):

3.18 Teacher: Okay let us look at all the angles at our disposal. If we add $A E C$ and $F C E$ ?

3.19 Lebogang and Lesiba: It's 180.

3.20 Teacher: This means that those co-interior angles are supplementary. If we have the co-interior angles that are supplementary, what can we say about the opposite lines?

3.21 Lebogang: Ohoo, then opposite sides are parallel.

3.22 Lesiba: Ah! Sir, this means we need two pairs of opposite sides that are parallel.

3.23 Sipho: And we can find the other pair by [interrupted by Lebogang].

3.24 Lebogang: We can find other co-interior angles.

3.25 Lesiba: Yes, write.

3.26 Lebogang: [writes $\angle B+\angle C=180]$ then co-interior angles are supplementary.

3.27 Sipho: Then we must also give a pair of opposite sides.

3.28 Lesiba: It will be line $A B$ and line $D C$.

3.29 Teacher: Okay good, so since we have two pairs of opposite sides that are parallel, what can we generally say?

3.30 Lebogang: This means we can say that this is a parallelogram.

3.31 Sipho: Yes, write there.

In this vignette, the source of intervention was the teacher who was a participant-observer in this study. His intervention was intentional because the question he asked drew the learners' attention to co-interior angles. The intention was explicit because he pointed learners to $\angle A E C$ and $\angle F C E$. This was an intentional intervention in which the teacher, abandoning Sipho's thinking of working at an inner layer, directed the learners to fold back and collect at an inner layer. This kind of intervention provided the teacher with an opportunity to explicitly model his thought processes to learners. This helped learners to modify and rebuild their construct of parallel lines while at the image having level of understanding - applying a mental construct about a co-interior angles of parallel lines without first developing it. This use of the sum of co-interior angles that are supplementary in order to show that lines are parallel resulted in an effective form of folding back, namely collecting at an inner layer.

In line 3.24 Lebogang intentionally intervened and suggested that the same way of thinking be followed to prove that the other pair of opposite sides are parallel. In line 3.26 she writes that $\angle B+\angle C=180$. This is another instance where Lebogang seemed not to distinguish between what needs to be proved and rudimentary facts about parallelograms that have become intuitive. Unfortunately, none of the group members, including the teacher, corrected this because Lebogang's unsubstantiated finding ultimately helped the learners to 
prove that the quadrilateral $A B C D$ is a parallelogram. In this instance, the learners failed to use existing understanding to work at an inner layer. This is more so because Sipho's suggestion that they work at an inner layer (line 3.16) had caused a discontinuity. Nonetheless, the outcome of folding back was effective; it resulted in learners' growth in how to apply the knowledge that if the sum of co-interior angles of two lines is $180^{\circ}$ then the lines are parallel. This happened although the group seemed unaware of the need to explore and explain when solving geometric problems - a difficulty that was also highlighted by Jones (2002).

\section{Summary}

The analysis of the extracts presented in this article show that folding back supported geometric reasoning. This support was also evident in instances where there were discontinuities during the act of folding back. This was the case in Extract 2 and Extract 3 where the discontinuity was overcome when learners were sources of the intervention through critical sharing of ideas and reflections. In Extract 3 the discontinuity was overcome when the teacher was the source of the intervention regardless of whether it was explicit or not for resolving the geometry task. We therefore claimed that teachers' interventions that are not explicit encourage learners' self-awareness of the limitations of their current understanding, without prescribing a solution.

Effective folding back also happened when the form of folding back did not result in developed ideas that learners were working on. For example, the algebraic error committed in Extract 2 while learners were working outside the topic of geometry did not affect the outcome of folding back negatively. By the same token, in Extract 3 the outcome of folding back in geometric reasoning was positive: learners worked with a rudimentary fact when it was required that they provide proof while working at an inner layer using existing understanding. Working with rudimentary facts is not in line with the notion of folding back which provides learners with an opportunity to build a connected understanding of mathematical concepts through returning and thickening their earlier existing images (e.g. Martin \& Towers, 2016)

\section{Conclusions}

In this article we pursued the question: how does folding back support learners' geometric reasoning? We were guided by Pirie and Kieren's (1994) dynamic theory for the growth of mathematical understanding and Martin's (2008) elaboration of Pirie and Kieren's process of folding back to track two groups of learners from one Grade 10 class. The data revealed two critical factors that contributed to effective folding back: the learners' critical sharing of ideas, and the teacher's intentional intervention.

\section{Learners' critical sharing of ideas}

Learners' self-questioning and reflections on their thinking in response to questions posed by peers offered them opportunities to revise, modify and extend their initial ideas and ultimately build a connected understanding through folding back (Extract 1 and Extract 2). Using Martin and Towers's (2016) words, they managed to return to and thicken their earlier existing images. This was evident when learners justified their geometric actions through using geometric properties relevant to a particular task. As they attempted to justify their thinking they reverted to folding back between the different layers of understanding. It was also a collaborative learning environment that encouraged the learners to be interested not only in their own geometry conceptual development but in their peers as well.

\section{Teacher's intentional intervention}

It was the nature of the teacher's interventional decisions that led learners to fold back (Extract 2 and Extract 3). The teacher asked questions that alerted learners to be explicit in their geometric met-befores (Tall, 2008) in order to arrive at the solution. From Martin and Towers's (2016) argument, the teacher did not ignore problematic met-befores and thus encouraged effective folding back and at the same time allowed thickening to occur. Thus, through following learners' reasoning path the teacher was able to observe learners' current layers of understanding and how they were used to further develop their understanding.

\section{Acknowledgements}

Samuel.M. acknowledges a sponsorship grant from the Research Office of the University of Limpopo through the internal Research Chair in Quality Teaching and Learning.

\section{Competing interests}

The authors declare that they have no financial or personal relationships that may have inappropriately influenced them in writing this article.

\section{Authors' contributions}

Samuel.M. carried out the research project as a master's student supervised by K.C. and Satsope.M. was the cosupervisor. Samuel.M.'s initial draft of the manuscript was reconceptualised by Satsope.M. and K.C. and thereafter Satsope.M. wrote the introduction, theoretical framework, research methodology, quality criteria, ethical consideration and conclusion sections. K.C. wrote the analysis, aligned and enriched it guided by the theoretical framework. I.K. played a role of a critical reader, improved on the logical flow of ideas and filled in the gaps to thus improve the quality of the manuscript.

\section{References}

Battista, M.T. (2007). The development of geometric and spatial thinking. In F.K Lester (Ed.), Second handbook of research on mathematics teaching and learning (pp. 843-908). Charlotte, NC: Information Age Publishing.

Bitsch, V. (2005). Qualitative research: A grounded theory example and evaluation criteria. Journal of Agribusiness, 23(1), 75-91. Retrieved from http://purl.umn. edu/59612 
Brown, M., Jones, K., Taylor, R., \& Hirst, A. (2004). Developing geometrical reasoning. In I. Putt, R. Faragher, \& M. McLean (Eds.), Proceedings of the 27th Annua Conference of the Mathematics Education Research Group of Australasia
(pp. 127-134). Sydney: MERGA. Retrieved from https://www.merga.net.au/ documents/RP132004.pdf

Budi, M. (2011, July). Traditional teaching about angles compared to an active learning approach that focuses on students' skills in seeing, measuring and reasoning, including the use of dynamic geometry software: Differences in achievement. Paper presented at the International Seminar and the Fourth National Conference
on Mathematics Education, Yogyakarta, Indonesia. Retrieved from http://eprints. on Mathematics Education, Yogyal
uny.ac.id/917/1/P\%20-\%204.pdf

Cohen, L., Manion. L., \& Morrison, K. (2007). Research methods in education. London: Routledge. https://doi.org/10.4324/9780203029053

Creswell, J.W. (2012). Qualitative inquiry and research design: Choosing among five approaches (2nd edn.). Thousand Oaks, CA: Sage.

Delgado, M.L., Codes, M., Monterrubio, M.C., \& Astudillo, M.T. (2014). The concep of numerical series. A study using the Pirie and Kieren model focused on the mechanism 'folding back'. Research Progress in Mathematics Education, 6, 25-44.

Department of Basic Education. (2011). Curriculum and assessment policy statement: Further education and training phase. Mathematics, Grade 10-12. Pretoria: DBE.

Evans, R. (2007). Proof and geometric reasoning. Mathematics Teaching Incorporating Micromath, 201, 38-41.

Fetisov, A.I., \& Dubnov, Y.S. (2012). Proof in geometry: With 'mistakes in geometric proofs'. Mineola, NY: Dover Publications.

Fujita, T., Yutaka, K., Kunimune, S., \& Jones, K. (2014). Open tasks in Japanese textbooks: The case of geometry for lower secondary school. In K. Jones, C. Bokhove, G. Howson, \& F. Lianghuo (Eds.), Proceedings of the International Conference on Mathematics Textbook Research and Development (pp. 233-238). Southampton: University of Southampton. Retrieved from https://eprints soton. ac.uk/374809/1/ICMT-2014_proceedings150331.pdf

Gal, H., \& Linchevski, L. (2010). To see or not to see: Analyzing difficulties in geometry from the perspective of visual perception. Educational Studies in Mathematics, 74(2), 163-183. https://doi.org/10.1007/s10649-010-9232-y

Guba, E.G., \& Lincoln, Y.S. (1989). Fourth generation evaluation. Newbury Park, CA Sage.

Gunhan, B.C. (2014). A case study on the investigation of reasoning skills in geometry. South African Journal of Education, 34(2), 1-19. https://doi. org/10.15700/201412071156

Hanna, G., \& De Villiers, M. (2008). ICMI study 19: Proof and proving in mathematics education. ZDM: The International Journal on Mathematics Education, 40(2) 329-336. https://doi.org/10.1007/s11858-008-0073-4

Jones, K. (2002). Issues in the teaching and learning of geometry. In: L. Haggarty (Ed) Aspects of teaching secondary mathematics (pp. 121-139). London: Routledge. Aspects of teaching secondary
https://doi.org/10.1002/tl.62

Kim, J.H. (2015). Understanding narrative inquiry: The crafting and analysis of stories as research. Los Angeles, CA: Sage.

Mabotja, K.S. (2017). An exploration of folding back in improving Grade 10 students reasoning in geometry. Unpublished master's thesis, University of Limpopo, Mankweng, South Africa. Retrieved from http://hdl.handle.net/10386/1805
Martin, L.C. (1999). The nature of the folding back phenomenon within the PirieKieren theory for the growth of mathematical understanding and the associated implications for teachers and learners of mathematics. Doctoral dissertation, University of Oxford, Oxford, England.

Martin, L.C. (2008). Folding back and the dynamical growth of mathematical understanding: Elaborating the Pirie-Kieren theory. The Journal of Mathematical Behavior, 27(1), 64-85. https://doi.org/10.1016/j.jmathb.2008.04.001

Martin, L.C., LaCroix, L., Fownes, L., Industry, B.C., \& Improvement, S. (2005). Fractions in the workplace: Folding back and the growth of mathematical understanding. International Group for the Psychology of Mathematics Education, 305.

Martin, L.C., \& Towers, J. (2016). Folding back, thickening and mathematical met-befores. The Journal of Mathematical Behavior, 43, 89-97. https://doi org/10.1016/j.jmathb.2016.07.002

Naidoo, J. (2013). Does social class influence learner reasoning in geometry? Global Journal of Medical Research, 13(3), 1-9. Retrieved from http:// medicalresearchjournal.org/index.php/GJMR/article/view/356/274

National Council of Teachers of Mathematics. (2002). Overview of principles and standards for school mathematics: Principles for school mathematics. Reston, VA: NCTM.

Ndlovu, M., \& Mji, A. (2012). Pedagogical implications of students' misconceptions about deductive geometric proof. Acta Academica, 44(3), 175-205. Retrieved from http://hdl.handle.net/10520/EJC131994

Panaoura, G., \& Gagatsis, A. (2009). The geometrical reasoning of primary and secondary school students. In V. Durand-Guerrier, S. Soury-Lavergne, \& F. Arzarello (Eds.), The Sixth Conference of the European Society for Research in Mathematics Education: Working Group 5, Geometrical Thinking (pp. 746-755). Lyon: Institut National de Recherchepédagogique. Retrieved from http://ife.enslyon.fr/publications/edition-electronique/cerme6/cerme6.pdf

Pirie, S., \& Kieren, T. (1994). Growth in mathematical understanding: How can we characterise it and how can we represent it? Educational Studies in Mathematics, 26(2), 165-190. https://doi.org/10.1007/BF01273662

Pirie, S., \& Martin, L. (2000). The role of collecting in the growth of mathematical understanding. Mathematics Education Research Journal, 12(2), 127-146. https://doi.org/10.1007/BF03217080

Polkinghorne, D.E. (1995). Narrative configuration in qualitative analysis. In J.A. Hatch \& R. Wisniewski (Eds.), Life history as narrative (pp. 5-23). London: The Falmer Press. https://doi.org/10.1080/0951839950080103

Powell, A.B., Francisco, J.M., \& Maher, C.A. (2003). An analytical model for studying the development of learners' mathematical ideas and reasoning using videotape data. The Journal of Mathematical Behavior, 22(4), 405-435. https://doi. org/10.1016/j.jmathb.2003.09.002

Steffe, L.P., \& Thompson, P.W. (2000). Teaching experiment methodology: Underlying principles and essential elements. In R. Lesh \& A.E. Kelly (Eds.), Handbook of research design in mathematics and science education (pp. 267-306). Hillsdale, NJ: Erlbaum.

Tall, D. (2008). The transition to formal thinking in mathematics. Mathematics Education Research Journal, 20(2), 5-24. https://doi.org/10.1007/BF03217474

Wright, V. (2014). Frequencies as proportions: Using a teaching model based on Pirie and Kieren's model of mathematical understanding. Mathematics Education Research Journal, 26(1), 101-128. https://doi.org/10.1007/s13394-014-0118-7 